\title{
CONVOLUTIONS OF CONTINUOUS MEASURES AND SUMS OF AN INDEPENDENT SET
}

\author{
JAMES MICHAEL RAGO ${ }^{1}$
}

\begin{abstract}
Let $E$ be a compact independent subset of an l.c.a. group $G ; \mu_{1}, \cdots, \mu_{n+1}$ continuous regular bounded Borel measures on $G$; and $k_{1}, \cdots, k_{n}$ integers. Let $k_{i} \times E=\left\{k_{i} x \mid x \in E\right\}$. We prove (1) $\mu_{1} * \cdots * \mu_{n+1}\left(k_{1} \times E+\cdots+k_{n} \times E\right)=0$ (the proof is a combinatorial argument).

As a corollary of (1) we obtain (2) if $H$ is any closed nondiscrete subgroup of $G$, then the intersection of $H$ with the group generated by $E$ has zero $H$-Haar measure.
\end{abstract}

1. In [2, Theorem 2], Hartman and Ryll-Nardzewski showed neatly that if $\mu_{1}$ and $\mu_{2}$ are continuous bounded measures on $T$, the circle group, and $E \subset T$ is a compact independent subset of $T$, then $\mu_{1} * \mu_{2}(E)=0$. In a more complicated manner, Salinger and Varopoulos proved this result for any metrizable group $G$ in [4, Theorem 1]. We prove a generalization of this theorem which holds for an arbitrary nondiscrete l.c.a. group $G$.

We denote by $Z$ the set of all integers, $Z^{+}=\{k \in Z \mid k>0\}$. A set $E \subset G$ is independent if the equation $k_{1} e_{1}+\cdots+k_{n} e_{n}=0, k_{i} \in Z, e_{i} \in E$, yields $k_{i} e_{i}=0$ for all $i=1, \cdots, n$ or the $e_{i}$ 's are not distinct. Note that an independent set may contain 0 . We set $k \times E=\{k e \mid e \in E\}$, and $n E=$ $\left\{e_{1}+\cdots+e_{n} \mid e_{i} \in E\right\}$ for $k \in Z, n \in Z^{+} ; E_{1}+E_{2}=\left\{e_{1}+e_{2} \mid e_{1} \in E_{1}, e_{2} \in E_{2}\right\}$. $M(G)$ is the set of bounded regular Borel measures on $G$.

Here is our main result.

THEOREM. If $G$ is a locally compact abelian group, $E \subset G$ a compact independent subset, $\mu_{1}, \cdots, \mu_{n+1} \in M(G)$ positive continuous measures, $k_{i} \in Z, i=1, \cdots, n$, and $x_{0} \in G$, then

$$
\mu_{1} * \cdots * \mu_{n+1}\left(k_{1} \times E+\cdots+k_{n} \times E-x_{0}\right)=0 .
$$

At the end of the proof of the theorem, we will derive the following corollary:

COROLlary. If $G$ is an l.c.a. group, $H \subset G$ is a closed nondiscrete subgroup with Haar measure $h, x_{0} \in G$, and $E \subset G$ is compact independent, then $h\left[\left(G p E-x_{0}\right) \cap H\right]=0$, where $G p E=\bigcup_{n=1}^{\infty} n(E \cup-E)$.

Received by the editors June 12, 1973.

AMS (MOS) subject classifications (1970). Primary 43A05.

Key words and phrases. Compact independent set, Haar measure.

${ }^{1}$ This work formed a part of the author's Ph.D. dissertation, which was written at Northwestern University under the supervision of Colin C. Graham. 
2. Proof of Theorem. The proof is by induction. If $n=1$, we follow the proof of [2, Theorem 2]. Suppose $k_{1}=1$. By definition,

$$
\mu_{1} * \mu_{2}(E)=\int_{G} \mu_{1}(E-x) d \mu_{2}(x) .
$$

For $x_{1} \neq x_{2}$, suppose there is a fixed element $y_{0} \in E-x_{1} \cap E-x_{2}$; then $y_{0}=e_{0}-x_{1}=f_{0}-x_{2}$, where $e_{0}, f_{0} \in E$ are fixed. Hence,

$$
\begin{aligned}
& x_{1}+y_{0}=e_{0} ; \\
& x_{2}+y_{0}=f_{0} .
\end{aligned}
$$

If $y \in E-x_{1} \cap E-x_{2}$ is arbitrary, then $y=e-x_{1}=f-x_{2}$ for certain $e, f \in E$, so

$$
\begin{aligned}
& x_{1}+y=e ; \\
& x_{2}+y=f .
\end{aligned}
$$

The equation (symbolically written) $(2.1)-(2.2)=(2.3)-(2.4)$ yields $e_{0}-f_{0}=e-f$. Since either $e_{0}$ or $f_{0}$ is nonzero (otherwise $x_{1}=x_{2}$ ), the independence of $E$ requires one of the following four possibilities: $e_{0}=$ $e, e_{0}=f, f_{0}=e$, or $f_{0}=f$. By (2.3) and (2.4), these imply

$$
y=e_{0}-x_{1}, y=f_{0}-x_{1}, y=e_{0}-x_{2} \text {, or } y=f_{0}-x_{2}
$$

respectively. Since $e_{0}, f_{0}, x_{1}$, and $x_{2}$ are fixed, this shows

$$
\operatorname{card}\left[E-x_{1} \cap E-x_{2}\right] \leqq 4
$$

and $\mu_{1}\left(E-x_{1} \cap E-x_{2}\right)=0$, by continuity of $\mu_{1}$. (Since $e_{0}-x_{1}=f_{0}-x_{2}=y_{0}$, we actually have card $\leqq 3$.) The set of $x$ with $\mu_{1}(E-x)>0$ is then countable, since it is easy to see that otherwise $\left\|\mu_{1}\right\|=\infty$. Hence by (1) and the continuity of $\mu_{2}$, we have $\mu_{1} * \mu_{2}(E)=0$. If $x_{0} \in G, \mu_{1} * \mu_{2}\left(E-x_{0}\right)=$ $\left[\left(\delta_{x_{0}} * \mu_{1}\right) * \mu_{2}\right]=0$, where $\delta_{x_{0}}$ is the point mass at $x_{0}$. Finally, in the case $k_{1} \neq 1, k_{1} \times E$ is also compact independent, so the Theorem holds for $n=1$.

We now suppose the Theorem holds for $n-1$ and all $k_{1}, \cdots, k_{n-1} \in Z$; we show it holds for $n$. Let $\mu_{1}, \cdots, \mu_{n+1}, k_{1}, \cdots, k_{n}$ and $x_{0}$ be given. Suppose

$$
\mu_{1} * \cdots * \mu_{n+1}\left(k_{1} \times E+\cdots+k_{n} \times E\right)=\delta>0 .
$$

Lemma. E may be written as the finite disjoint union of Borel sets $E_{i}$, $i=1, \cdots, p$, such that for some choice of integers $i_{1}, \cdots, i_{n}, 0 \leqq i_{1}<\cdots<$ $i_{n} \leqq p$, we have

$$
\mu_{1} * \cdots * \mu_{n+1}\left(k_{1} \times E_{i_{1}}+\cdots+k_{n} \times E_{i_{n}}\right)>0 .
$$


Proof of Lemma. We first set $k=\sum_{1}^{n}\left|k_{i}\right|$. By the inductive hypothesis of the Theorem, for each choice of integers $l_{1}, \cdots, l_{n-1}$, we have

$$
\mu_{1} * \cdots *\left(\mu_{n} * \mu_{n+1}\right)\left(\sum_{1}^{n-1} l_{i} \times E\right)=0 .
$$

Containing each set $\sum_{1}^{n-1} l_{i} \times E$ there is, therefore, by the regularity of $\mu_{1} * \cdots * \mu_{n+1}$, an open set $U_{l_{1}, \cdots, l_{n-1}}$ with $\sum l_{i} \times E \subset U_{l_{1}, \cdots, l_{n-1}}$, and

$$
\mu_{1} * \cdots * \mu_{n+1}\left(U_{l_{1}, \ldots, l_{n-1}}\right)<\delta / A,
$$

where $A$ is the number of possible choices of $l_{1}, \cdots, l_{n-1}$ with $\sum_{1}^{n-1}\left|l_{i}\right| \leqq k$. (For example, $A \leqq(2 k+1)^{n-1}$.)

For each $U_{l_{1}, \cdots, l_{n-1}}$, there is a relatively compact neighborhood $V$ of 0 such that

$$
\sum_{1}^{n-1} l_{i} \times E+k \bar{V} \subset U_{l_{1}}, \cdots, l_{n-1},
$$

by compactness. Taking (finite) intersections when necessary, we may assume (6) holds with one $V$ for all choices of $\left(l_{1}, \cdots, l_{n-1}\right)$ with $\sum_{1}^{n-1}\left|l_{i}\right| \leqq k$.

Since $E$ is compact, it is covered by finitely many (say $p$ ) neighborhoods $\left(e_{i}+V\right) \cap E$, with $e_{i} \in E, i=1, \cdots, p(p$ depending on $V)$. Define $E_{1}=$ $\left(e_{1}+\bar{V}\right) \cap E$,

$$
E_{i}=\left(e_{i}+\bar{V}\right) \cap E \backslash\left(E_{1} \cup \cdots \cup E_{i-1}\right), \quad i=2, \cdots, p,
$$

so the $E_{i}$ 's are disjoint Borel sets with union $E$. Each set of the form

$$
F=k_{1} \times E_{i_{1}}+\cdots+k_{n} \times E_{i_{n}}, \quad 1 \leqq i_{1}, \cdots, i_{n} \leqq p,
$$

is Borel. We claim that at least one of the sets of form (7), with $i_{1}<\cdots<i_{n}$ all distinct, has strictly positive $\mu_{1} * \cdots * \mu_{n+1}$-measure. This will prove the lemma.

Indeed, consider any set of form (7) with at least two subscripts equal, say $i_{1}=i_{2}$. It may then be written

$$
\begin{aligned}
F= & k_{1} \times E_{i_{1}}+k_{2} \times E_{i_{1}}+k_{3} \times E_{i_{3}}+\cdots+k_{n} \times E_{i_{n}} \\
\subset & k_{1} \times\left(e_{i_{1}}+\bar{V}\right)+k_{2} \times\left(e_{i_{1}}+\bar{V}\right)+k_{3} \times\left(e_{i_{3}}+\bar{V}\right) \\
& +\cdots+k_{n} \times\left(e_{i_{n}}+\bar{V}\right) \\
\subset & \left(k_{1}+k_{2}\right) \times E+\cdots+k_{n} \times E+k \bar{V} \\
\subset & U_{\left(k_{1}+k_{2}, k_{3}, \cdots, k_{n}\right)}
\end{aligned}
$$

by (6). Hence the union of such sets (with two subscripts equal) is contaimed in the union of the $(A)$ sets $U_{l_{1}, \cdots, l_{n-1}}$, and thus has $\mu_{1} * \cdots *$ $\mu_{n+1}$-measure less than $\delta$, by (5). This completes the proof of the Lemma. 
For convenience we will assume, relabelling if necessary, that $i_{1}=$ $1, \cdots, i_{n}=n$. We write

$$
\begin{aligned}
\mu_{1} * \cdots * \mu_{n+1} & \left(\sum_{1}^{n} k_{i} \times E_{i}\right) \\
& =\int \mu_{1} * \cdots * \mu_{n}\left(\sum k_{i} \times E_{i}-x\right) d \mu_{n+1}(x) .
\end{aligned}
$$

We claim that

$$
x_{1} \neq x_{2} \Rightarrow \mu_{1} * \cdots * \mu_{n}\left(\sum_{1}^{n} k_{i} \times E_{i}-x_{1} \cap \sum_{1}^{n} k_{i} \times E_{i}-x_{2}\right)=0 .
$$

Then as in the proof of the case $n=1$, we have $\mu_{1} * \cdots * \mu_{n}\left(\sum k_{i} \times E_{i}-x\right)=$ 0 except for perhaps countably many $x$, and we obtain, by (8),

$$
\mu_{1} * \cdots * \mu_{n+1}\left(\sum_{1}^{n} k_{i} \times E_{i}\right)=0,
$$

contradicting (4) and hence (3). We will thus obtain

$$
\mu_{1} * \cdots * \mu_{n+1}\left(\sum_{1}^{n} k_{i} \times E\right)=0
$$

To prove the claim, suppose there is a fixed $y_{0} \in\left(\sum k_{i} \times E_{i}-x_{1}\right) \cap$ $\left(\sum k_{i} \times E_{i}-x_{2}\right)$. Then

$$
\begin{aligned}
& x_{1}+y_{0}=k_{1} e_{1}^{0}+\cdots+k_{n} e_{n}^{0}, \\
& x_{2}+y_{0}=k_{1} f_{1}^{0}+\cdots+k_{n} f_{n}^{0},
\end{aligned}
$$

$e_{i}^{0}, f_{i}^{0} \in E_{i}$ fixed. Given an arbitrary $y \in\left(\sum k_{i} \times E_{i}-x_{1}\right) \cap\left(\sum k_{i} \times E_{i}-x_{2}\right)$, we have

$$
\begin{aligned}
& x_{1}+y=k_{1} e_{1}+\cdots+k_{n} e_{n}, \\
& x_{2}+y=k_{1} f_{1}+\cdots+k_{n} f_{n},
\end{aligned}
$$

$e_{i}, f_{i} \in E_{i}$. For at least one value of $i$, say $i=j$, we must have $k_{j} e_{j} \neq k_{j} f_{j}$, since otherwise $x_{1}=x_{2}$. Hence the equation denoted by $(11.1)-(11.2)=$ (11.3)-(11.4) and the disjointness of the $E_{i}$ 's with the independence of $E$ yields

$$
k_{j} e_{j}^{0}-k_{j} f_{j}^{0}-k_{j} e_{j}+k_{j} f_{j}=0 .
$$

Since one of the first two terms of (12) must be nonzero, we must have (again by independence) that one of $e_{j}$ or $f_{j}$ must equal $e_{j}^{0}$ or $f_{j}^{0}$, the latter two being fixed. Then by (11.3) and (11.4), either $y=k_{j} e_{j}^{0}+\sum_{i \neq j} k_{i} e_{i}-x_{1}$, $y=k_{j} f_{j}^{0}+\sum_{i \neq j} k_{i} e_{i}-x_{1}, y=k_{j} e_{j}^{0}+\sum_{i \neq j} k_{i} f_{i}-x_{2}$, or $y=k_{j} f_{j}^{0}+\sum_{i \neq j} k_{i} f_{i}-x_{2}$; 
hence we have

$$
y \in \bigcup_{s=1,2}\left(k_{j} e_{j}^{0}+\sum_{i \neq j} k_{i} \times E_{i}-x_{s}\right) \cup \bigcup_{s=1,2}\left(k_{j} f_{j}^{0}+\sum_{i \neq j} k_{i} \times E_{i}-x_{s}\right) .
$$

Since $y$ is arbitrary, $\left(\sum k_{i} \times E_{i}-x_{1}\right) \cap\left(\sum k_{i} \times E_{i}-x_{2}\right)$ is contained in a finite union (over $j=1, \cdots, n$ ) of sets of the above form; however, by the inductive hypothesis,

$$
\begin{aligned}
& \mu_{1} * \cdots * \mu_{n+1}(\left.k_{j} e_{j}^{0}+\sum_{i \neq j} k_{i} \times E_{i}-x_{s}\right) \\
&=\left(\mu_{1} * \mu_{2}\right) * \cdots * \mu_{n+1}\left(\sum_{i \neq j} k_{i} \times E_{i}-\left(x_{s}-k_{j} e_{j}^{0}\right)\right)=0,
\end{aligned}
$$

(there are $n$-convolutions and $n-1$-summands); $s=1,2$, and similarly for $f_{j}^{0}$. Hence the claim, and (10), are proved. (We note it is not generally possible to have card $\left[\sum k_{i} \times E_{i}-x_{1} \cap \sum k_{i} \times E_{i}-x_{2}\right]<\infty$; for example, choose $E$ infinite and fix $x_{1}, x_{2} \in E$; then $E \subset E+E-x_{1} \cap E+E-x_{2}$.)

Finally,

$$
\begin{aligned}
\mu_{1} * \cdots * \mu_{n+1} & \left(\sum_{i=1}^{n} k_{i} \times E-x_{0}\right) \\
& =\left(\delta_{x_{0}} * \mu_{1}\right) * \cdots * \mu_{n+1}\left(\sum_{i=1}^{n} k_{i} \times E-x_{0}\right)=0,
\end{aligned}
$$

and the Theorem is proved. Q.E.D.

Note that the requirement that $\mu_{i}$ is positive may be lifted by passing to the total variation measure.

If $G$ is a compact nondiscrete group with Haar measure $h, E \subset G$ compact independent, and $G p(E)=\bigcup_{n=1}^{\infty} n(E \cup-E)$, then our Theorem implies at once that $h(G p E)=0$. Indeed, $h$ is a bounded, continuous, idempotent measure, and thus annihilates $\sum_{i=1}^{n} k_{i} \times E$ for all $n$ and $k_{i}$. Our Corollary is a generalization of this, due to Graham [1] (who strengthened Rudin $[3,5.3 .6])$, giving a proof considerably simpler than Graham's.

3. Proof of Corollary. Given a closed set $S \subset G$, define $\left.h\right|_{S}(A)=$ $h(A \cap S \cap H)$ for all $A \subset G, A$ Borel. In particular, we may write $\left.h\right|_{H}=h$, and thus consider $h$ as a (possibly unbounded) measure on $G$. We claim that if $S \subset H$ and $A \subset G$ are compact sets, then setting $B=A-n S$ for any $n \in Z^{+}$, we obtain

$$
\left.\left.\left.h\right|_{B} * h\right|_{S} * \cdots * h\right|_{S}(A)=h(S)^{n} h(A),
$$


where there are $n$-convolutions of $\left.h\right|_{S}$. Indeed,

$$
\begin{aligned}
\left.\left.h\right|_{B} * h\right|_{S} * & \left.\cdots * h\right|_{S}(A) \\
& =\left.\left.\left.\int h\right|_{B}\left(A-x_{1}-\cdots-x_{n}\right) d h\right|_{S}\left(x_{1}\right) \cdots d h\right|_{S}\left(x_{n}\right) .
\end{aligned}
$$

Since $A-x_{1}-\cdots-x_{n} \subset A-n S=B$ for $x_{i} \in S, i=1, \cdots, n$,

$$
\left.h\right|_{B}\left(A-x_{1}-\cdots-x_{n}\right)=h\left(A-x_{1}-\cdots-x_{n}\right)=h(A)
$$

because $x_{i} \in S \subset H$ implies $\left(A-x_{1}-\cdots-x_{n}\right) \cap H=A \cap H-x_{1}-\cdots-x_{n}$. Thus,

$$
\begin{aligned}
\left.\int h\right|_{B}\left(A-x_{1}-\right. & \left.\cdots-x_{n}\right)\left.\left.d h\right|_{S}\left(x_{1}\right) \cdots d h\right|_{S}\left(x_{n}\right) \\
& =\left.\left.\int h(A) d h\right|_{S}\left(x_{1}\right) \cdots d h\right|_{S}\left(x_{n}\right) \\
& =h(S)^{n} h(A),
\end{aligned}
$$

and the claim is proved.

Suppose now that $E$ and $k_{1}, \cdots, k_{n}$ are given; we fix $W \subset H$ to be any relatively compact neighborhood in $H$, and set $A=k_{1} \times E+\cdots+k_{n} \times$ $E-x_{0}, S=\bar{W}, B=A-n S$ as above. Since $\left.h\right|_{B S}$ and $\left.h\right|_{S}$ are then bounded continuous measures on $G$, our Theorem implies

$$
\left.\left.\left.h\right|_{B^{2}} * h\right|_{S} * \cdots * h\right|_{S}(A)=0,
$$

where there are $n$-convolutions of $\left.h\right|_{S}$. However, (13) implies that $h(S)^{n} h(A)=0$. Since $\bar{W}=S$, then $h(S) \neq 0$ and it follows that $h(A)=0$. Thus $h\left[\left(G p E-x_{0}\right) \cap H\right]=0$. Q.E.D.

I would like to express my gratitude to the referee for his helpful remarks and criticisms.

\section{REFERENCES}

1. C. Graham, Compact independent sets and Haar measure, Proc. Amer. Math. Soc. 36 (1972), 578-582.

2. S. Hartman and C. Ryll-Nardzewski, Quelques résultats et problèmes en algèbre des mesures continues, Colloq. Math. 22 (1971), 271-277. MR 44 \#1993.

3. W. Rudin, Fourier analysis on groups, Interscience Tracts in Pure and Appl. Math., no. 12, Interscience, New York, 1962. MR 27 \#2808.

4. D. L. Salinger and N. Th. Varopoulos, Convolutions of measures and sets of analyticity, Math. Scand. 25 (1969), 5-18. MR 41 \#8930.

Department of Mathematics, Northwestern University, Evanston, Illinois 60201

Current address: 6507 North Kedzie Avenue, Chicago, Illinois 60645 